\title{
Localization And Distribution Of Vibrio fischeri Symbionts In Euprymna tasmanica Light Organ Affect Symbiosis
}

\author{
Vinod Nair, Soumitra Ghoshroy and Michele Nishiguchi
}

Department of Biology and Electron Microscopy Laboratory

New Mexico State University, Las Cruces, NM 88003-88001

Most members of sepiolid squids (Mollusca: Cephalopoda) (Fig.1) harbors luminescent, symbiotic bacteria in specialized light organ complexes. Juvenile squids hatch axenically, following which they acquire symbiotic bacteria from the surrounding seawater. Bacteria that colonize the juvenile squids are housed in channels or crypts (Fig.2) of the bilobed light organ [1].Axenic juveniles have a pair of ciliated epithelial appendages (CEA) on either side of the light organ complex (Fig.3a), which helps entrain seawater containing bacteria over the pores leading into the light organ. Successful colonization by Vibrio symbionts causes apoptotic regression (Fig. 3b) of the CEA [2]. Despite the nature of this environmentally transmitted symbiosis, there is a high degree of host-symbiont specificity. Infection by interspecific $V$. fischeri symbionts exhibit preferential colonization of the light organ with the native $V$. fischeri strains [3]. Studies on localization and distribution of $V$. fischeri strains in the light organ were executed to determine if placement of the symbiotic bacteria in the light organ complex determine host specificity. The morphological variation of pili types produced by $V$. fischeri strains was investigated to address their involvement in host specificity. The goal of this study is to understand features that render native strains more competent.

For the localization and distribution experiments, juvenile squid co-infected with native and nonnative strains of $V$. fischeri was fixed in LR white resin, sectioned using MT2B Sorvall ultramicrotome and visualized using a Hitachi H-7000 TEM. The native strains of $V$. fischeri were transformed with GFP expressing plasmids. Immunolabeling for anti-GFP with $12 \mathrm{~nm}$ colloidal gold helped differentiate the native bacterial strains from the non- native $V$. fischeri strains lacking GFP plasmids. Bacterial pili were visualized using $2 \%$ uranyl acetate as a negative stain.

Previous study has demonstrated an increase in microvillar density up to four folds in sepiolid light organ following bacterial colonization of the crypts [4]. In addition, we observed that the brush border epithelial (BBE) cells intimately associated with the bacteria became short and stubby, loosing their microvillus like appearance (Fig4 a, b). Native strains were found closely associated with the BBE and non-native strains were observed to be more centrally distributed in the crypt (Fig. 5). This central distribution and lack of adhesion to the light organ cells may be the result of expulsion of non-native bacteria during venting cycles leaving behind the native strains that may be better at adhering to the host epithelia. Host specificity demonstrated by $V$. fischeri strains may be attributed to the morphologically diverse pili produced by the bacteria (Fig.6 a, b, c). Bacteria have been known to alter the nature of pili based on environmental cues. The diverse pili morphology exhibited by symbiotic $V$. fischeri suggests their possible role in adhesion to the host tissue thus rendering specificity to this association.

References

[1] M. J. McFall-Ngai and E. G. Ruby, Science (1991) 254(5037): 1491-4.

[2] K. L. Visick and M. J. McFall-Ngai, J. Bacteriol (2000) 182(7): 1779-87

[3] M.K. Nishiguchi et al. Appl. Environ. Microbiol (1998) (9): 3209-13

[4] L.H. Lamarq and M. J. McFall-Ngai, Infect. Immun. (1998) 66(2): 777-785 

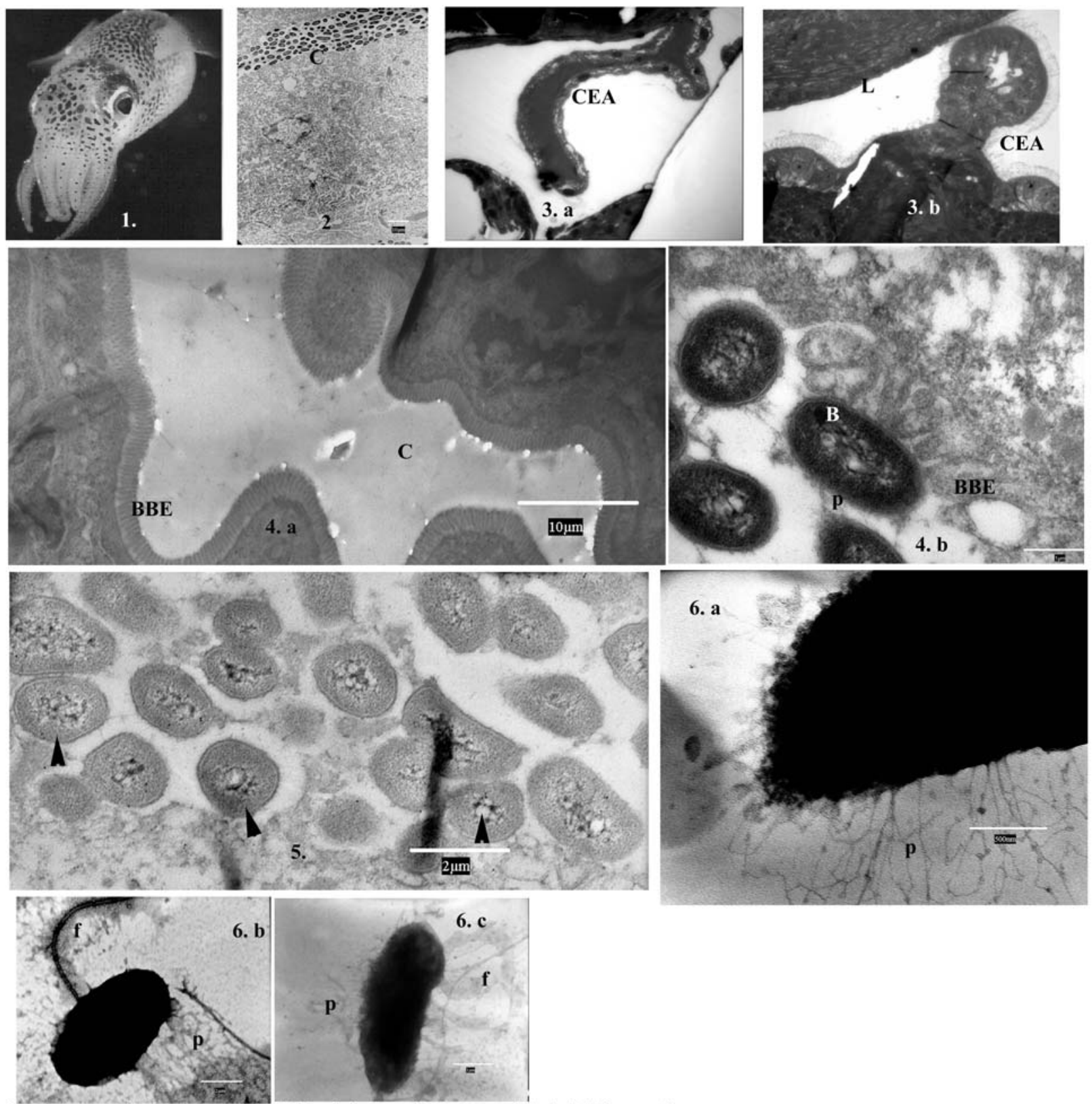

Fig.1 An adult E. scolopes individual: $(\sim 5 \mathrm{~cm})$ photo credit: $A . W$. Omerod

Fig. 2 TEM image of $\mathrm{V}$. fischeri in light organ crypt $(c)$. (bar $=10 \mu \mathrm{m})$

Fig. 3 (a) Cross sectional view of aposymbiotic squid light organ showing CEA

(b) Cross sectional view of symbiotic squid light organ showing regressed CEA and lens (L)

Fig. 4 (a) TEM image of crypt (c) showing microvillus like BBE (bar $=10 \mu \mathrm{m})$

(b) TEM image demonstrating bacterial pili and their interaction with BBE $($ bar $=1 \mu \mathrm{m})$

Fig. 5 TEM image showing the distribution of native and non-native bacteria in the crypt.

Immunolabeled cells shown with arrowheads. $($ bar $=\mathbf{2} \mu \mathrm{m})$

Fig. 6 Negatively stained $V$. fischeri strains showing pili (p) and flagella (f).

(a) ES114 strain $($ bar $=500 \mathrm{~nm})$, (b) ES915 strain $(\mathrm{bar}=2 \mu \mathrm{m}),(\mathrm{c})$ ETB1B $(\mathrm{bar}=1 \mu \mathrm{m})$ 